\title{
ON THE PERFORMANCE OF OPTICAL FLOW ROUTERS EMPLOYING WAVELENGTH CONVERSION
}

\author{
J. J. He and D. Simeonidou \\ Department of Electronic Systems Engineering, University of Essex, Colchester, UK \\ \{jhea, dsimeo\}@essex.ac.uk
}

\begin{abstract}
In optical flow-routing networks, packet flows are transmitted through optical flow routers. Featuring the increased good-throughput, optical flow routers have been proposed as a substitute to optical packet routers for the future optical Internet. This paper studies the optical flow routers using wavelength conversion and optical buffering as the contention resolution. A detailed analysis employing Markov process is provided in order to investigate the performance of optical flow routers and to compare with optical packet routers. Results show that wavelength conversion is a promising technique in improving the optical flow routers' performance. Up to $99 \%$ good-throughput can be achieved in an optical flow router with 10 wavelength conversions and 60 buffers under a traffic load per wavelength of 2 . On the other hand our results suggest that the good-throughput of optical packet routers cannot be improved by wavelength conversion.
\end{abstract}

Key words: Flow routing, wavelength conversion, optical buffering, good-throughput

\section{INTRODUCTION}

Internet traffic is exceeding the traditional telephony traffic and becoming dominant in the telecommunication network due to the widely used TCP/IP protocol suite [1]. At the same time the Internet is demanding more and more network capacity. As a result, optical networks with large capacity have been developed for the future Internet. With the advance of the state-of-art optical technology, optical packet switching seems to be an efficient technique for the optical networks to support the bursty Internet traffic with various quality-of-service requirements [2].

\footnotetext{
A. Bianco et al. (eds.), Next Generation Optical Network Design and Modelling

(C) Springer Science+Business Media New York 2003
} 
Optical packet switching inherits a packet contention problem, which arises when multiple packets arrive at an output port at the same time. To reduce the packet loss probability for optical packet routers, optical buffering, wavelength conversion, and multi-path routing have been introduced, where the effectiveness of using these techniques has been reported. As a result, the end system may receive out-of-order packets due to the packets of the same flow being transmitted through different wavelength channels or fibres by wavelength conversion or multi-path routing. The arriving times of the packets may also be different. Studies have shown that a large amount of out-of-order packets will drive the higher-layer protocols in the end system, such as TCP, to misbehave [3], causing the end system to generate more bursty Internet traffic and hence more network resources will be required to resolve the contentions.

Recently optical flow routing technology has been proposed, with which a flow of packets is routed and switched as a whole [4]. Analyses under both continuous and bursty traffic conditions show that optical flow router (OFR) with optical buffering is superior to optical packet-router (OPR). The amount of the packets of the corrupted flows that contain out-of-order packets decreases by deploying OFR. For example, when 60 buffers are used, $90.2 \%$ packets belong to the corrupted flows after the traffic passing an OPR with a load of 2 , while this figure reduces to $56.2 \%$ after the traffic passing an OFR.

Nevertheless, if the traffic goes through several such OFRs in series, the percentage of the total packets composing non-corrupted flows (i.e. the good-throughput) can be very small. For instance, if the traffic passes 3 OFRs, only $8.4 \%$ packets will be in order given the previous condition. Obviously, this will limit the deployment of OFRs. To address this limitation, wavelength conversion is considered in OFRs in addition to optical buffering.

Wavelength conversion is an essential technique in improving the blocking probability in optical circuit-switching networks [5] and in reducing the packet loss probability in optical packet-switching networks [6]. For example, the packet loss probability can decrease from $10^{-6}$ to $10^{-11}$ by employing 2 wavelength conversions in an optical packet router [6].

In this paper, we will focus on the analytical investigation for the performance of an OFR with wavelength conversion and optical buffering. As a comparison, wavelength-converted OPR will also be studied. In the next section the traffic model and the discarding operations for the buffers of the routers are described. Detailed analysis is presented in Section 3. Section 4 gives the results and discussions, following by the concluding remarks in Section 5. 


\section{MODELS}

\subsection{Traffic model}

Previous studies showed that the performance characteristics of OFRs and OPRs with optical buffering under the bursty traffic condition are similar to that under the continuous traffic condition [7,8]. Thus the continuous traffic model will be considered, making the analysis simpler.

As in [7], for each wavelength channel we assume a Poisson packet arrival rate $\lambda^{\prime}$, an exponential distribution for the packet transmission time with the mean of $1 / \mu$ time slots, and a truncated geometric distribution with $\mathrm{q}$ for the number of packets in a flow. A first-in-first-out output buffering is considered. The buffer depth of each wavelength is $B$ in time slot. Thus the load per wavelength is $\lambda / \mu$, with $(\lambda / \mu)>1$ indicating the occurrence of the contentions. $\lambda$ is the actual packet arrival rate for each wavelength channel, which is calculated as $\lambda=\lambda^{\prime}\left[1-q \cdot(1-q)^{\mathrm{L}}\right] . \mathrm{L}$ is the maximum number of packets in a flow, which is related to $B$ and $\mu$ as $(L / \mu) \leq B$. Given the number of wavelengths in a fibre being $\mathrm{n}_{\mathrm{w}}$ the packet arrival rate of the fibre is $\Lambda=n_{\mathrm{w}} \cdot \lambda$.

\subsection{Discarding operations for buffers}

The discarding operations considered for the wavelength-converted OFRs are flow discard (FD) and early-flow discard (EFD). With FD, a flow will be stored or transmitted in the available wavelength with the lowest index as illustrated in Fig. 1. As shown, three flows from different wavelengths are arriving at the same time at an output fiber with three wavelength channels. The flow from the first wavelength is transmitted via wavelength $\mathrm{c} 1$; the one from the second wavelength is wavelength converted and stored in the buffer of wavelength $\mathrm{c} 1$; and the one from the last wavelength is wavelength converted and transmitted via wavelength $c 2$ due to not-enough buffer space in wavelength $\mathrm{c} 1$. Thus wavelength $\mathrm{c} 3$ can be reserved for the coming traffic, and the buffer space is created as large as possible, resulting in the maximum good-throughput for the router.

With EFD, a threshold $k$ for the buffer occupancy will be set, beyond which no new flows can be accepted. Thus an arriving flow will seek a buffer space in the next available wavelength if the current one exceeds $k$. This scenario is described in Fig. 2. As the same as that in Fig. 1, three flows are arriving at the same time. The first flow is transmitted via wavelength $\mathrm{cl}$ as in FD. After that the buffer occupancy of wavelength c1 exceeds the threshold, therefore the flow from the second wavelength is transmitted via wavelength $\mathrm{c} 2$. The one from the third wavelength is wavelength converted 
and stored in wavelength $\mathrm{c} 2$ since the buffer occupancy of $\mathrm{c} 2$ has not exceeded the threshold at that time. Wavelength $\mathrm{c} 3$ is reserved for the coming traffic.

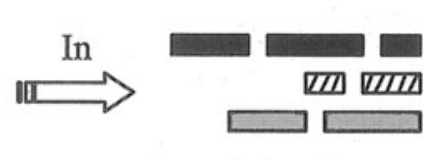

arriving flows

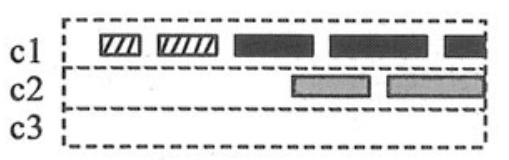

flows in buffers

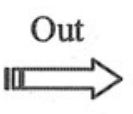

Figure 1. Buffering flows in a wavelength-converted OFR router with flow discard.

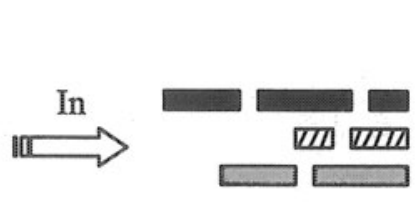

arriving flows

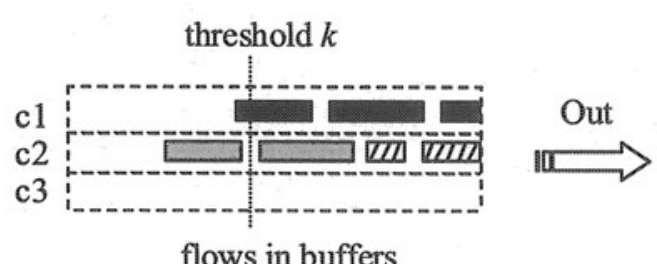

flows in buffers

Figure 2. flows in a wavelength-converted OFR router with early flow discard

For wavelength-converted OPRs, packet discard (PD) is used. PD is similar to FD in OFRs, except that the entity to be dealt with is "packet" instead of "flow". Thus each packet in a flow will be routed and switched independently. When contention occurs the conflicting packet will be buffered or wavelength converted. The sequence of the packets in the same flow cannot be guaranteed.

3.

\subsection{Steady-state analysis of buffer occupancy}

For a fibre channel with output buffering and wavelength conversion, the place of the occupied buffer can be addressed as $(m, i)$, where $m$ is the index of the wavelength channel $\left(1 \leq \mathrm{m} \leq \mathrm{n}_{\mathrm{w}}\right)$ and $\mathrm{i}$ is the buffer being occupied for that particular wavelength channel $(0 \leq \mathrm{i} \leq \mathrm{B})$. By applying the discarding operations of FD, EFD and PD, Markov chains for the buffer occupancy can be formed. To conduct the steady-state analysis of the buffer occupancy, we introduce a system mode parameter 1 , which indicates the wavelength channel is discarding traffic $(1=1)$ or not $(1=0)$. Therefore for 
the occupancy of the buffer that locates at wavelength channel $\mathrm{m}$ and buffer $i$, we can use $(m, i, 1)$ to express the state of the buffer.

\subsubsection{Deriving the steady-state probability of buffer occupancy for OPR with PD}

For OPR with $\mathrm{PD}$, each arriving packet will be discarded only when it fails to find buffer space in all the $n_{w}$ wavelength channels. Thus the queuing system only has one discarding status, that is $\left(\mathrm{n}_{\mathrm{w}}, \mathrm{B}, 1\right)$. The state-transitionrate diagram for OPR with PD is depicted in Fig. 3. Let $P_{m, i, 1}$ be the steadystate probability of having $\mathrm{m}$ transmission channels being occupied, the buffer occupancy be $i$ at the $m$-th wavelength and the system be in mode 1 , we can easily derive the following set of equations for the steady-state system:

$$
\begin{aligned}
& \Lambda \mathrm{P}_{1,0,0}=\mu \mathrm{P}_{1,1,0} \\
& (\Lambda+m \mu) P_{m, 0,0}=\Lambda P_{m-1, B, 0}+m \mu P_{m, 1,0} \quad 2 \leq m \leq n_{w} \\
& (\Lambda+m \mu) \mathrm{P}_{\mathrm{m}, \mathrm{i}, 0}=\Lambda \mathrm{P}_{\mathrm{m}, \mathrm{i}-1,0}+\mathrm{m} \mu \mathrm{P}_{\mathrm{m}, \mathrm{i}+1,0} \\
& 1 \leq \mathrm{m} \leq \mathrm{n}_{\mathrm{w}} \text { and } 1 \leq \mathrm{i} \leq \mathrm{B}-2 \\
& \text { or, } 1 \leq \mathrm{m} \leq \mathrm{n}_{\mathrm{w}}-1 \text { and } \mathrm{i}=\mathrm{B}-1 \\
& \left(\Lambda+\mathrm{n}_{\mathrm{w}} \mu\right) \mathbb{P}_{\mathrm{n}_{\mathrm{w}}, \mathrm{B}-1,0}=\Lambda \mathbb{P}_{\mathrm{n}_{\mathrm{w}}, \mathrm{B}-2,0}+\mathrm{n}_{\mathrm{w}} \mu\left(\mathbb{P}_{\mathrm{n}_{\mathrm{w}}, \mathrm{B}, 0}+\mathbb{P}_{\mathrm{n}_{\mathrm{w}}, \mathrm{B}, 1}\right) \\
& (\Lambda+m \mu) P_{m, B, 0}=\Lambda P_{m, B-1,0}+(m+1) \mu P_{m+1,0,0} \quad 1 \leq m \leq n_{w}-1 \\
& \left(\Lambda+\mathrm{n}_{\mathrm{w}} \mu\right) \mathbf{P}_{\mathrm{n}_{\mathrm{w}}, \mathrm{B}, 0}=\Lambda \mathbf{P}_{\mathrm{n}_{\mathrm{w}}, \mathrm{B}-1,0} \\
& \mathrm{n}_{\mathrm{w}} \mu \mathrm{P}_{\mathrm{n}_{\mathrm{w}}, \mathrm{B}, 1}=\Lambda \mathrm{P}_{\mathrm{n}_{\mathrm{w}}, \mathrm{B}, 0}
\end{aligned}
$$

and,

$$
\mathbf{P}_{\mathrm{n}_{\mathrm{W}}, \mathrm{B}, 1}+\sum_{\mathrm{m}=1}^{\mathrm{n}_{\mathrm{W}}} \sum_{\mathrm{i}=0}^{\mathrm{B}} \mathbf{P}_{\mathrm{m}, \mathrm{i}, 0}=1
$$

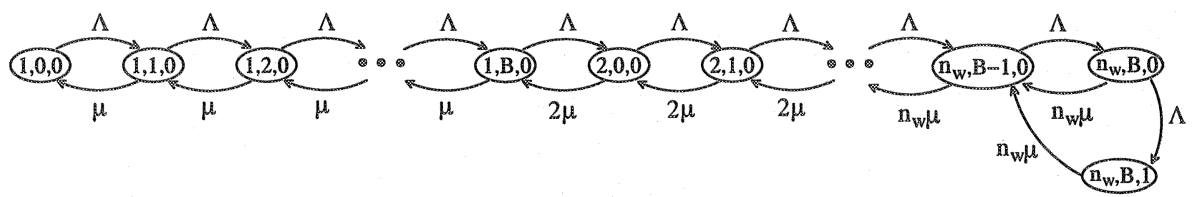

Figure 3. State transition diagram for the buffer queue of a PD router with wavelength conversion.

\subsubsection{Deriving the steady-state probability of buffer occupancy for OFR with FD}

In the wavelength-converted OFR with $F D$, an arriving flow is discarded only after it fails to find buffers for the whole flow in all the wavelength 
channels. Therefore from the packet's point of view, when the head packet of a flow arrives, the system will allocate the resources in the available wavelength channel with the lowest index to all the packets in the flow. The state-transition-rate diagram for this scenario is shown in Fig. 4, from which the following set of equations can be derived when the system reaches the steady state.

$$
\begin{aligned}
& \Lambda \mathrm{P}_{1,0,0}=\mu \mathrm{P}_{1,1,0} \\
& \mathrm{eP}_{1,0,1}=\mu \mathrm{P}_{1,1,1} \\
& (\Lambda+\mathrm{m} \mu) \mathrm{P}_{\mathrm{m}, 0,0}=\mathrm{m} \mu \mathrm{P}_{\mathrm{m}, \mathrm{i}+1,0}+\mathrm{e}\left(\mathrm{P}_{\mathrm{m}-1, \mathrm{~B}, 0}+\mathrm{P}_{\mathrm{m}-1, \mathrm{~B}, 1}\right) \\
& +\sum_{i=1}^{B} \sum_{1=0}^{1} a_{i} P_{m}-1, i, 1 \quad 2 \leq m \leq n_{w} \\
& (\mathrm{e}+\mathrm{m} \mu) \mathrm{P}_{\mathrm{m}, 0,1}=\mathrm{m} \mu \mathrm{P}_{\mathrm{m}, 1,1} \quad 2 \leq \mathrm{m} \leq \mathrm{n}_{\mathrm{W}} \\
& \left(\mathrm{a}_{1}+\mathrm{b}_{1}+\mathrm{m} \mu\right) \mathrm{P}_{\mathrm{m}, 1,0}=\mathrm{m} \mu \mathrm{P}_{\mathrm{m}, 2,0}+\Lambda \mathrm{P}_{\mathrm{m}, 0,0}+\mathrm{eP}_{\mathrm{m}, 0,1} \quad 1 \leq \mathrm{m}<\mathrm{n}_{\mathrm{W}} \\
& \left(\mathrm{a}_{1}+\mathrm{c}_{1}+\mathrm{m} \mu\right) \mathrm{P}_{\mathrm{m}, 1,1}=\mathrm{m} \mu \mathrm{P}_{\mathrm{m}, 2,1} \quad 1 \leq \mathrm{m}<\mathrm{n}_{\mathrm{W}} \\
& \left(a_{i}+b_{i}+m \mu\right) P_{m, i, 0}=b_{i-1} P_{m, i-1,0}+m \mu P_{m, i+1,0}+c_{i-1} P_{m, i-1,1} \\
& 1 \leq \mathrm{m}<\mathrm{n}_{\mathrm{W}} \text { and } 1<\mathrm{i}<\mathrm{B} \\
& \left(\mathrm{a}_{\mathrm{i}}+\mathrm{c}_{\mathrm{i}}+\mathrm{m} \mu\right) \mathrm{P}_{\mathrm{m}, \mathrm{i}, 1}=\mathrm{m} \mu \mathrm{P}_{\mathrm{m}, \mathrm{i}+1,1} \quad 1 \leq \mathrm{m}<\mathrm{n}_{\mathrm{W}} \text { and } 1<\mathrm{i}<\mathrm{B} \\
& (\mathrm{m} \mu+\mathrm{e}+\mathrm{d}) \mathrm{P}_{\mathrm{m}, \mathrm{B}, 0}=\mathrm{b}_{\mathrm{B}-1} \mathrm{P}_{\mathrm{m}, \mathrm{B}-1,0}+(\mathrm{m}+1) \mu \mathrm{P}_{\mathrm{m}+1,0,0}+\mathrm{c}_{\mathrm{B}-1} \mathrm{P}_{\mathrm{m}, \mathrm{B}-1,1} \\
& 1 \leq \mathrm{m}<\mathrm{n}_{\mathrm{W}} \\
& (\mathrm{m} \mu+\mathrm{e}) \mathrm{P}_{\mathrm{m}, \mathrm{B}, 1}=\mathrm{dP}_{\mathrm{m}, \mathrm{B}, 0}+(\mathrm{m}+1) \mu \mathrm{P}_{\mathrm{m}+1,0,1} \quad 1 \leq \mathrm{m}<\mathrm{n}_{\mathrm{W}} \\
& \left(\mathrm{n}_{\mathrm{W}} \mu+\mathrm{a}_{1}+\mathrm{b}_{1}\right) \mathrm{P}_{\mathrm{n}_{\mathrm{W}}, 1,0}=\Lambda \mathrm{P}_{\mathrm{W}}, 0,0+\mathrm{n}_{\mathrm{w}} \mu \mathrm{P}_{\mathrm{n}_{\mathrm{W}}, 2,0}+\mathrm{eP}_{\mathrm{n}_{\mathrm{W}}, 0,1} \\
& \left(\mathrm{n}_{\mathrm{W}} \mu+\mathrm{c}_{1}\right) \mathrm{P}_{\mathrm{n}_{\mathrm{W}}, 1,1}=\mathrm{a}_{1} \mathbf{P}_{\mathrm{n}_{\mathrm{W}}, 1,0+\mathrm{n}_{\mathrm{W}}} \mu \mathrm{P}_{\mathrm{n}_{\mathrm{W}}, 2,1} \\
& \left(\mathrm{n}_{\mathrm{w}} \mu+\mathrm{a}_{\mathrm{i}}+\mathrm{b}_{\mathrm{i}}\right) \mathrm{P}_{\mathrm{n}_{\mathrm{W}}, \mathrm{i}, 0}=\mathrm{b}_{\mathrm{i}-1} \mathrm{P}_{\mathrm{n}_{\mathrm{W}}, \mathrm{i}-1,0}+\mathrm{n}_{\mathrm{W}} \mu \mathrm{P}_{\mathrm{n}_{\mathrm{W}}, \mathrm{i}+1,0}+\mathrm{c}_{\mathrm{i}-1} \mathrm{P}_{\mathrm{n}_{\mathrm{W}}, \mathrm{i}-1,1} \\
& 1<\mathrm{i}<\mathrm{B} \\
& \left(\mathrm{n}_{\mathrm{W}} \mu+\mathrm{c}_{\mathrm{i}}\right) \mathrm{P}_{\mathrm{n}_{\mathrm{W}}, \mathrm{i}, 1}=\mathrm{a}_{\mathrm{i}} \mathrm{P}_{\mathrm{n}_{\mathrm{W}}, \mathrm{i}, 0}+\mathrm{n}_{\mathrm{W}} \mu \mathrm{P}_{\mathrm{n}_{\mathrm{W}}, \mathrm{i}+1,1} \quad 1<\mathrm{i}<\mathrm{B}
\end{aligned}
$$

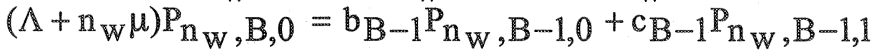

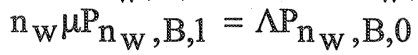

and,

$$
\sum_{\mathrm{m}=1}^{\mathrm{n}_{\mathrm{W}}} \sum_{\mathrm{i}=0}^{\mathrm{B}} \sum_{1=0}^{1} \mathrm{P}_{\mathrm{m}, \mathrm{i}, \mathrm{l}}=1
$$

Given $\Phi_{\text {ave }}$ being the average arriving rate of new flows in the fiber at time $t$, then the parameters for the above equation are that,

- $a_{i}$ is the rate of the arriving packet which is the head of a new flow with the number of packets larger than $(B-i) \cdot \mu$, so, 


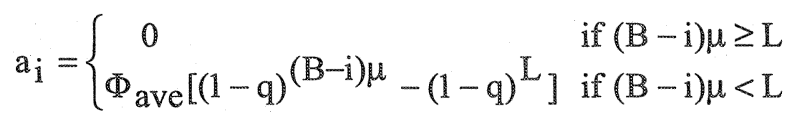

- $b_{i}$ is the rate of the arriving packet that is the head of a new flow with the number of packets less than $(B-i) \cdot \mu$ or belongs to the flow of which some packets have already been in the buffer of a particular wavelength, thus,

$$
b_{i}=\Lambda-a_{i}
$$

- $\mathrm{c}_{\mathrm{i}}$ is the rate of the arriving packet which is the head of a new flow with the number of packets less than $(B-i) \cdot \mu$ or $L$ (whichever is smaller), thus,

$$
c_{i}=\left\{\begin{array}{cc}
\Phi_{\text {ave }}\left[1-(1-q)^{L}\right] & \text { if }(B-i) \mu \geq L \\
\Phi_{\text {ave }}\left[1-(1-q)^{(B-i) \mu}\right] & \text { if }(B-i) \mu<L
\end{array}\right.
$$

- $e$ is the rate of the arriving packet which is the head of a new flow with the number of packets less than $\mathrm{L}$, thus,

$$
e=\Phi_{\text {ave }}\left[1-(1-q)^{L}\right]
$$

- $\mathrm{d}$ is the rate of the next arrival, which is not the head of a new flow, we have,

$\mathrm{d}=\Lambda-\mathrm{e}$

$\Phi$ ave is found to be:

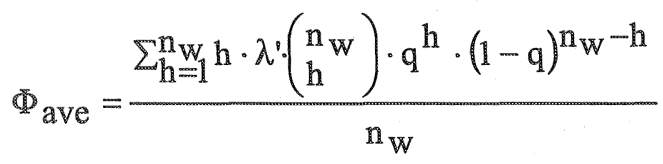



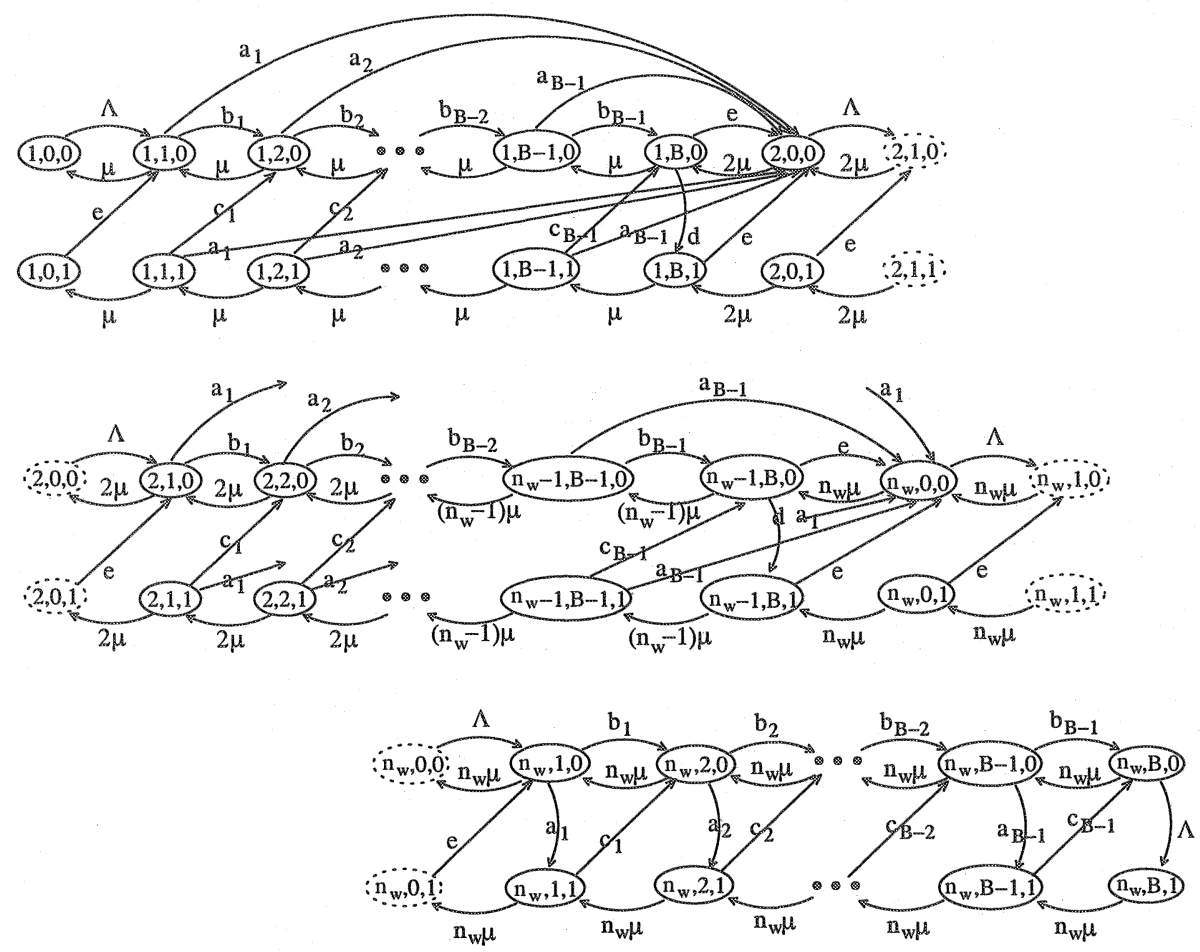

Figure 4 State transition diagram for the buffer queue of a FD router with wavelength conversion.

\subsubsection{Deriving the steady-state probability of buffer occupancy for OFR with EFD}

Similar to that with $\mathrm{FD}$, the wavelength-converted OFR with EFD will discard an arriving flow only when it fails to find buffers for the whole flow in all the wavelength channels. However the buffer in one wavelength channel will accept all flows till its occupancy exceeds a threshold. After that the buffer is not available to any arriving flows until the occupancy drops lower than the threshold. The state-transition-rate diagram is plotted in Fig. 5. We can derive the following set of equations for the system in the steady state. 


$$
\begin{aligned}
& \Lambda \mathrm{P}_{1,0,0}=\mu \mathrm{P}_{1,1,0} \\
& \mathrm{eP}_{1,0,1}=\mu \mathrm{P}_{1,2,1} \\
& (\Lambda+m \mu) P_{m, 0,0}=m \mu P_{m, 1,0}+e \sum_{i=k}^{B} \sum_{l=0}^{1} P_{m-1, i, 1} \quad 2 \leq m \leq n_{W} \\
& (\mathrm{e}+\mathrm{m} \mu) \mathrm{P}_{\mathrm{m}, 0,1}=\mathrm{m} \mu \mathrm{P}_{\mathrm{m}, 1,1} \quad 2 \leq \mathrm{m} \leq \mathrm{n}_{\mathrm{W}} \\
& (\Lambda+\mathrm{m} \mu) \mathrm{P}_{\mathrm{m}, \mathrm{i}, 0}=\Lambda \mathrm{P}_{\mathrm{m}, \mathrm{i}-1,0}+\mathrm{m} \mu \mathrm{P}_{\mathrm{m}, \mathrm{i}+1,0}+\mathrm{eP} \mathrm{P}_{\mathrm{m}, \mathrm{i}-1,1} \\
& 1 \leq \mathrm{m} \leq \mathrm{n}_{\mathrm{W}} \text { and } 1 \leq \mathrm{i}<\mathrm{k} \\
& (\mathrm{e}+\mathrm{m} \mu) \mathrm{P}_{\mathrm{m}, \mathrm{i}, 1}=\mathrm{m} \mu \mathrm{P}_{\mathrm{m}, \mathrm{i}+1,1} \quad 1 \leq \mathrm{m} \leq \mathrm{n}_{\mathrm{W}} \text { and } 1 \leq \mathrm{i}<\mathrm{k} \\
& (\mathrm{e}+\mathrm{m} \mu+\mathrm{d}) \mathrm{P}_{\mathrm{m}, \mathrm{k}, 0}=\Lambda \mathrm{P}_{\mathrm{m}, \mathrm{k}-1,0}+\mathrm{m} \mu \mathrm{P}_{\mathrm{m}, \mathrm{k}+1,0}+\mathrm{eP}_{\mathrm{m}, \mathrm{k}-1,1} \\
& 1 \leq \mathrm{m}<\mathrm{n}_{\mathrm{W}} \\
& (\mathrm{e}+\mathrm{m} \mu) \mathrm{P}_{\mathrm{m}, \mathrm{k}, 1}=\mathrm{m} \mu \mathrm{P}_{\mathrm{m}, \mathrm{k}+1,1} \quad 1 \leq \mathrm{m}<\mathrm{n}_{\mathrm{W}} \\
& (\mathrm{e}+\mathrm{m} \mu+\mathrm{d}) \mathrm{P}_{\mathrm{m}, \mathrm{i}, 0}=\mathrm{dP}_{\mathrm{m}, \mathrm{i}-1,0}+\mathrm{m} \mu \mathrm{P}_{\mathrm{m}, \mathrm{i}+1,0} \quad 1 \leq \mathrm{m}<\mathrm{n}_{\mathrm{W}} \text { and } \mathrm{k}<\mathrm{i}<\mathrm{B} \\
& (\mathrm{e}+\mathrm{m} \mu) \mathrm{P}_{\mathrm{m}, \mathrm{i}, 1}=\mathrm{m} \mu \mathrm{P}_{\mathrm{m}, \mathrm{i}+1,1} \quad 1 \leq \mathrm{m}<\mathrm{n}_{\mathrm{W}} \text { and } \mathrm{k}<\mathrm{i}<\mathrm{B} \\
& (\mathrm{e}+\mathrm{m} \mu+\mathrm{d}) \mathrm{P}_{\mathrm{m}, \mathrm{B}, 0}=\mathrm{dP}_{\mathrm{m}, \mathrm{B}-1,0}+(\mathrm{m}+1) \mu \mathrm{P}_{\mathrm{m}+1,0,0} \quad 1 \leq \mathrm{m}<\mathrm{n}_{\mathrm{W}} \\
& (\mathrm{e}+\mathrm{m} \mu) \mathrm{P}_{\mathrm{m}, \mathrm{B}, 1}=\mathrm{dP}_{\mathrm{m}, \mathrm{B}, 0}+(\mathrm{m}+1) \mu \mathrm{P}_{\mathrm{m}+1,0,1} \quad 1 \leq \mathrm{m}<\mathrm{n}_{\mathrm{W}} \\
& \left(\mathrm{e}+\mathrm{n}_{\mathrm{w}} \mu+\mathrm{d}\right) \mathrm{P}_{\mathrm{n}_{\mathrm{W}}, \mathrm{k}, 0}=\Lambda \mathbf{P}_{\mathrm{n}_{\mathrm{W}}, \mathrm{k}-1,0}+\mathrm{n}_{\mathrm{w}} \mu \mathrm{P}_{\mathrm{n}_{\mathrm{W}}, \mathrm{k}+1,0}+e \mathrm{P}_{\mathrm{n}_{\mathrm{W}}, \mathrm{k}-1,1} \\
& \mathrm{n}_{\mathrm{W}} \mu \mathrm{P}_{\mathrm{n}_{\mathrm{W}}, \mathrm{k}, 1}=\mathrm{n}_{\mathrm{W}} \mu \mathrm{P}_{\mathrm{n}_{\mathrm{W}}, \mathrm{k}+1,1}+\mathrm{eP}_{\mathrm{n}_{\mathrm{W}}, \mathrm{k}, 0}
\end{aligned}
$$

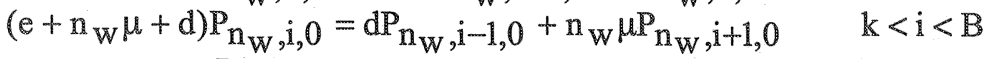

$$
\begin{aligned}
& \mathrm{n}_{\mathrm{W}} \mu \mathrm{P}_{\mathrm{n}_{\mathrm{W}}, \mathrm{i}, 1}=\mathrm{eP}_{\mathrm{n}_{\mathrm{W}}, \mathrm{i}, 0}+\mathrm{n}_{\mathrm{W}} \mu \mathrm{P}_{\mathrm{n}_{\mathrm{W}}, \mathrm{i}+1,1} \quad \mathrm{k}<\mathrm{i}<\mathrm{B}
\end{aligned}
$$

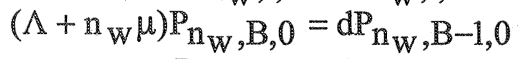

$$
\begin{aligned}
& \mathrm{n}_{\mathrm{W}} \mu \mathrm{P}_{\mathrm{n}_{\mathrm{W}}, \mathrm{B}, 1}=\Lambda \mathrm{P}_{\mathrm{n}_{\mathrm{W}}, \mathrm{B}, 0}
\end{aligned}
$$

and,

$$
\sum_{m=1}^{n_{w}} \sum_{i=0}^{B} \sum_{l=0}^{1} P_{m, i, 1}=1
$$

The definitions and expressions for e and $d$ are the same as that in the previous section. 

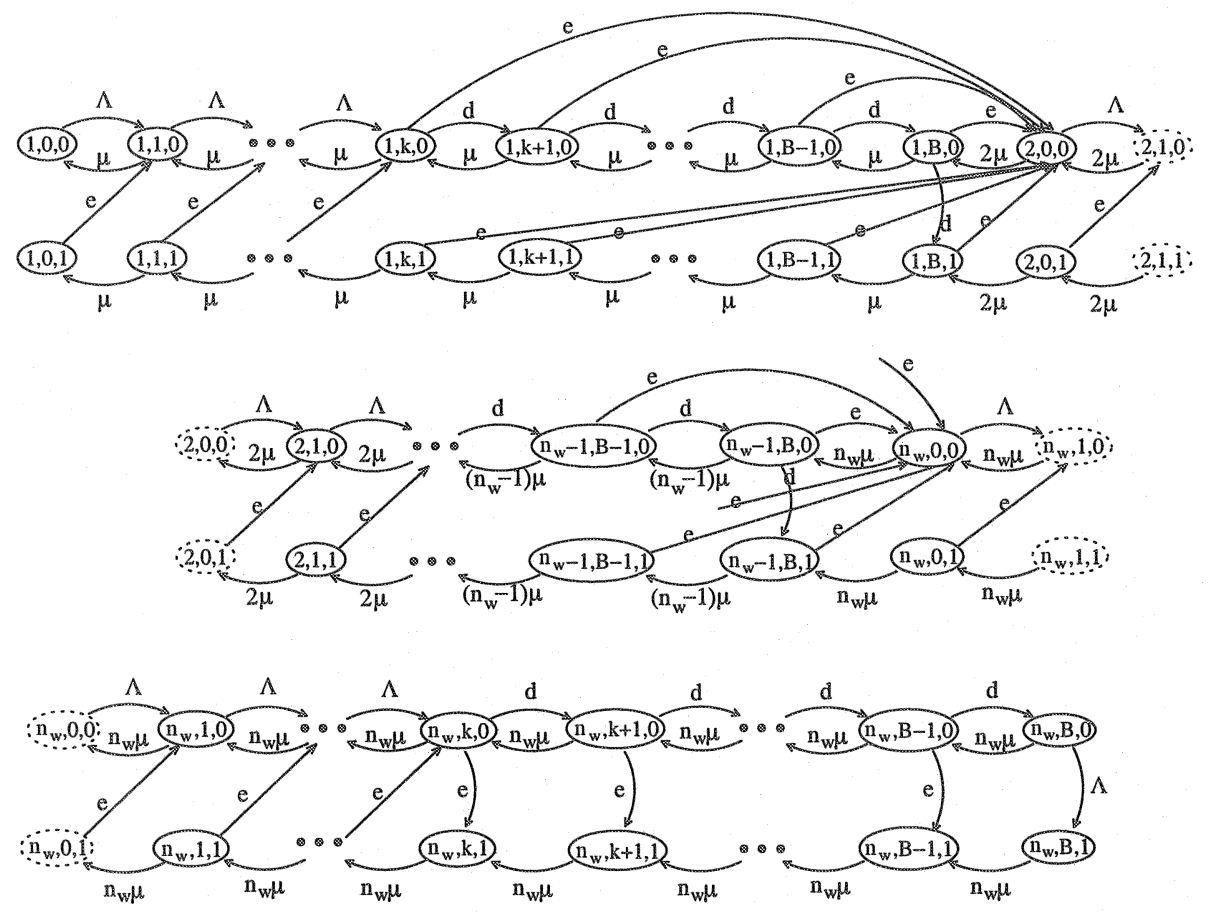

Figure 5 State transition diagram for the buffer queue of EFD router with wavelength conversion.

\subsection{Good-throughput}

We will adopt the good-throughput as the performance metric. Goodthroughput $(G)$ is the ratio of the sum of the packets composing noncorrupted flows to the total packets arriving at the router's input [4]. It represents the percentage of the traffic that is successful transmitted by the router.

According to the definition, good-throughput can be written as [7]:

$$
\begin{aligned}
G & =\frac{\sum_{n=1}^{L} n \cdot P(w=n, v=1)}{\sum_{n=1}^{L} n \cdot P(w=n)} \\
& =\frac{\sum_{n=1}^{L} n \cdot P(w=n, v=1)}{\sum_{n=1}^{L} n \cdot \frac{q \cdot(1-q)^{n-1}}{\sum_{j=1}^{L} q \cdot(1-q)^{j}}}
\end{aligned}
$$

where $w$ is the random variable representing the length of a flow in terms of the number of packets; $\mathrm{V}$ is the random variable representing the 
transmission of a flow, with $\mathrm{v}=1$ indicating a successfully transmitted flow and $\mathrm{v}=0$ indicating a failed one.

For the probability of a flow with $\mathrm{n}$ packets to be successfully transmitted, it can be calculated as following,

$$
\mathrm{P}(\mathrm{w}=\mathrm{n}, \mathrm{v}=1)=\mathbb{P}(\mathrm{v}=1 \mid \mathrm{w}=\mathrm{n}) \cdot \mathrm{P}(\mathrm{w}=\mathrm{n}) \quad \mathrm{n} \geq 1
$$

The conditional probability $\mathrm{P}(\mathrm{v}=1 \mid \mathrm{w}=\mathrm{n})$ for the wavelength-converted queuing system can be written as:

$$
P(v=1 \mid w=n)=\sum_{m=1}^{n_{W}} \sum_{i=0}^{B} P(v=1 \mid w=n, R=m, Q=i) \cdot P(R=m, Q=i)
$$

where $P(R=m, Q=i)$ is the probability of $m$ wavelength channels being used and $i$ buffer being occupied in the $\mathrm{m}$-th channel. It is easy to derive that,

$$
P(R=m, Q=i)=P(m, i, 0)+P(m, i, 1)
$$

The value of $P(m, i, 0)$ and $P(m, i, 1)$ can be obtained by solving the steady-state-transition rate equation corresponding to the $\mathrm{PD}, \mathrm{FD}$ or $\mathrm{EFD}$ routers.

Let $S_{n, m, i}=P(v=1 \mid w=n, R=m, Q=i)$, it can be derived for $P D, F D$ and $E F D$ routers as following.

For $\mathrm{PD}$, let $\mathrm{S}_{\mathrm{n}, \mathrm{m}, \mathrm{i}}^{\mathrm{PD}}=\mathrm{P}(\mathrm{v}=1 \mid \mathrm{w}=\mathrm{n}, \mathrm{R}=\mathrm{m}, \mathrm{Q}=\mathrm{i})$.

- If $0 \leq i \leq B-n / \mu$ and $1 \leq m \leq n_{w}$, there will be enough buffer space to accommodate the flow, thus, $S_{n, m, i}^{P D}=1$;

- If $\mathrm{i}=\mathrm{B}$ and $1 \leq \mathrm{m}<\mathrm{n}_{\mathrm{w}}$, we have $\mathrm{S}_{\mathrm{n}, \mathrm{m}, \mathrm{i}}^{\mathrm{PD}}=1$;

- If $\mathrm{i}=\mathrm{B}$ and $\mathrm{m}=\mathrm{n}_{\mathrm{w}}$, then $\mathrm{S}_{\mathrm{n}, \mathrm{m}, \mathrm{i}}^{\mathrm{PD}}=0$;

- If $B-n / \mu+1 \leq i \leq B-1$ and $1 \leq m \leq n_{w}$, then $S_{n, m, i}^{P D}=(1-r) S_{n-1, m, i+1}^{P D}+r S_{n, m, i-1}^{P D}$, where $r=\mu / \lambda+\mu$ is the probability that a departure occurs before an arrival [7].

For $F D$, let $S_{n, m, i}^{F D}=P(v=1 \mid w=n, R=m, Q=i)$.

- If $0 \leq \mathrm{i} \leq \mathrm{B}-\mathrm{n} / \mu$ and $1 \leq \mathrm{m} \leq \mathrm{n}_{\mathrm{w}}, \mathrm{S}_{\mathrm{n}, \mathrm{m}, \mathrm{i}}^{\mathrm{FD}}=1$;

- If $\mathrm{i}=\mathrm{B}$ and $1 \leq \mathrm{m}<\mathrm{n}_{\mathrm{w}}, \mathrm{S}_{\mathrm{n}, \mathrm{m}, \mathrm{i}}^{\mathrm{FD}}=1$; 
- If $\mathrm{i}=\mathrm{B}$ and $\mathrm{m}=\mathrm{n}_{\mathrm{w}}, \mathrm{S}_{\mathrm{n}, \mathrm{m}, \mathrm{i}}^{\mathrm{FD}}=0$;

- If $B-n / \mu+1 \leq i \leq B$ and $m<n_{w}$, the arriving flow can admit to the buffer of the next wavelength channel, thus $S_{n, m, i}^{F D}=1$;

- However, if $B-n / \mu+1 \leq i \leq B$ and $m=n_{w}, S_{n, m, i}^{F D}=0$.

For EFD, the effect of the threshold should be considered. Let $\mathrm{S}_{\mathrm{n}, \mathrm{m}, \mathrm{i}}^{\mathrm{EFD}}=\mathrm{P}(\mathrm{v}=1 \mid \mathrm{w}=\mathrm{n}, \mathrm{R}=\mathrm{m}, \mathrm{Q}=\mathrm{i})$,

- If $\mathrm{i}<\mathrm{k}$ and $1 \leq \mathrm{m} \leq \mathrm{n}_{\mathrm{w}}, \mathrm{S}_{\mathrm{n}, \mathrm{m}, \mathrm{i}}^{\mathrm{EFD}}=\mathrm{S}_{\mathrm{n}, \mathrm{m}, \mathrm{i}}^{\mathrm{PD}}$;

- If $\mathrm{i} \geq \mathrm{k}$ and $1 \leq \mathrm{m}<\mathrm{n}_{\mathrm{w}}$, the flow will be accommodated in the buffer of the next wavelength channel, we have $\mathrm{S}_{\mathrm{n}, \mathrm{m}, \mathrm{i}}^{\mathrm{EFD}}=\mathrm{S}_{\mathrm{n}, \mathrm{m}+1,0}^{\mathrm{EFD}}$

- When $\mathrm{i} \geq \mathrm{k}$ and $\mathrm{m}=\mathrm{n}_{\mathrm{w}}$, the flow has to be discarded, therefore $\mathrm{S}_{\mathrm{n}, \mathrm{m}, \mathrm{i}}^{\mathrm{EFD}}=0$.

\section{RESULTS AND DISCUSSIONS}

According to the results obtained in the previous studies [7], the buffer depth $\mathrm{B}$ will be fixed at 60 and the threshold $\mathrm{k}$ for the buffer occupancy of $E F D$ routers will be at the half of the buffer depth, i.e. $k=30$. Other parameters are $\mu=1, \lambda=2$, and $L=10$, unless stated.

\subsection{Effect of wavelength conversion}

The effect of the number of wavelength conversions $n_{w}$ on the goodthroughput $G$ is studied firstly. The results are shown in Figs. $6 a$ and $6 b$ for FD, EFD and PD routers.

As can be seen, G for PD router is much lower than that for FD and EFD routers. In addition it doesn't change with the increase of $\mathrm{n}_{\mathrm{w}}$ for both the cases of $q=1 / 5$ and $q=1 / 8$, where $G$ is kept at $16.48 \%$ and $9.85 \%$ respectively. This is because the amount of packets that are converted to other wavelengths and thus affect the good-throughput depends on the load per wavelength. Although the packet loss probability can be reduced by using wavelength conversion in PD routers, the good-throughput or the quality of the transmitted traffic cannot be improved. Thus by using wavelength conversion in the intermediate OPRs the end system will still receive the data traffic containing massive out-of-sequence packets, which 
will in turn cause the Internet traffic more bursty and consequently degrade the performance of the data networks.

While for FD and EFD routers, $\mathrm{G}$ increases with $\mathrm{n}_{\mathrm{w}}$. For instance in Fig. $6 a$, when $q=1 / 5, G$ of $94.38 \%$ and $65 \%$ can be obtained with 10 wavelength conversions for FD and EFD routers respectively, comparing with $45.16 \%$ and $50 \%$ when no wavelength conversion is used. Furthermore up to $99 \%$ good-throughput for FD routers can be obtained for $q=1 / 8$. These results indicate that the quality of the transmitted traffic can be improved by using wavelength conversion in OFRs. In addition, G for FD routers is higher than that for EFD routers when wavelength conversion is used $\left(n_{w}>1\right)$. The higher the $n_{w}$ the bigger the difference. For example in Fig. $6 a$ the difference of $G$ between FD and EFD routers is $14.87 \%$ when $\mathrm{n}_{\mathrm{w}}=3$, while this difference increases to $29.3 \%$ for $\mathrm{n}_{\mathrm{w}}=10$. This is due to the low value of the threshold some flows cannot admit to the buffers of EFD routers. Analytical results show that the good-throughput of the wavelength-converted EFD routers increases with the threshold. Fig. 7 depicts the good-throughput $\mathrm{G}$ versus the threshold $\mathrm{k}$ for EFD routers with 4 wavelength conversions under various wavelength loads. As shown G imposes a big increase when $\mathrm{k}$ approaches to the buffer depth. This reveals that for the wavelength-converted EFD routers larger threshold values should be used, which is not the case for the buffer-only EFD routers.

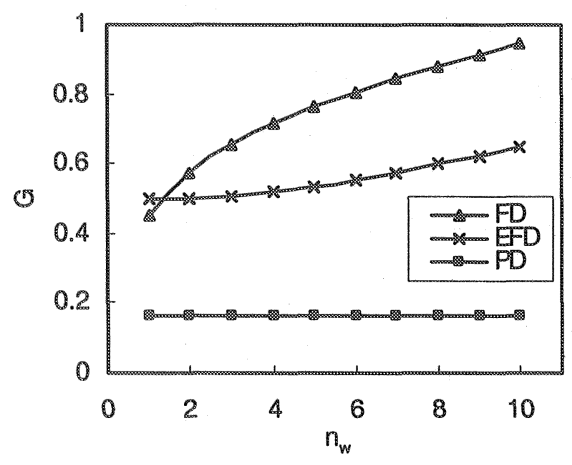

(a)

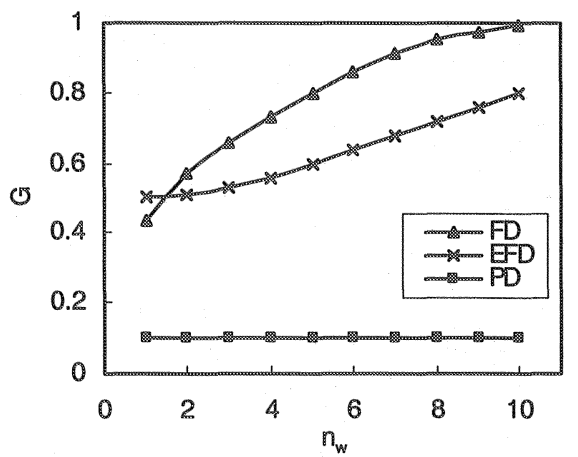

(b)

Figure 4. Good-throughput $\mathrm{G}$ vs. number of wavelength conversions $\mathrm{n}_{\mathrm{W}}$ for $\mathrm{FD}, \mathrm{EFD}$ and PD routers, $\lambda=2, \mu=1, L=10, B=60$, and $k=30$, (a) $q=1 / 5$; (b) $q=1 / 8$. 


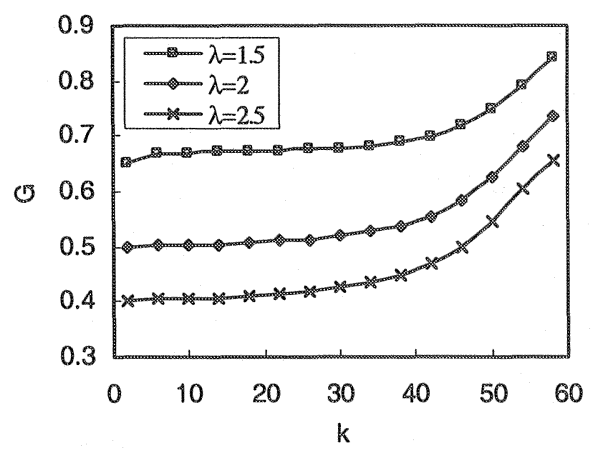

Figure 5. Good-throughput $\mathrm{G}$ vs. threshold $\mathrm{k}$ for $\mathrm{EFD}$ routers, $\mathrm{n}_{\mathrm{w}}=4, \mu=1, \mathrm{q}=1 / 5$, $\mathrm{L}=10, \mathrm{~B}=60$.

\subsection{Eifect of channel load}

Next we investigate the effect of varying the wavelength load $\lambda$ on the good-throughput $\mathrm{G} . \mathrm{n}_{\mathrm{w}}$ is kept at 4 . The results are shown in Figs. 8a and $8 \mathrm{~b}$ for $\mathrm{q}=1 / 5,1 / 8$ respectively. It can be seen that $\mathrm{G}$ decreases with the increase of $\lambda$, which is similar to the buffer-only case. Comparing Fig. 8a and Fig. $8 b$, with the increase of the mean flow length $1 / q, G$ of OFRs increases while $G$ of OPRs decreases. However $G$ for FD routers is always higher than that for EFD routers. It is believed that with a higher threshold value, $\mathrm{G}$ for $\mathrm{EFD}$ routers will be close to that for $\mathrm{FD}$ routers.

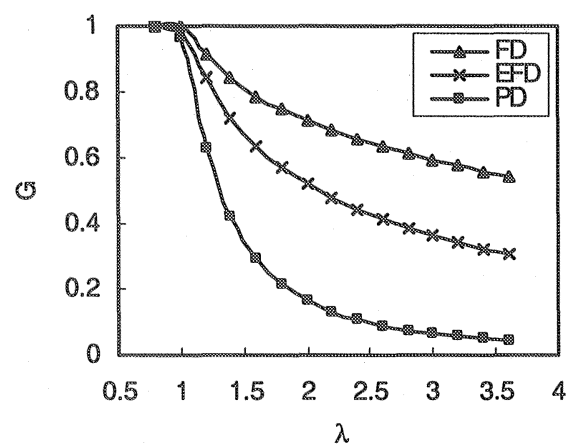

(a)

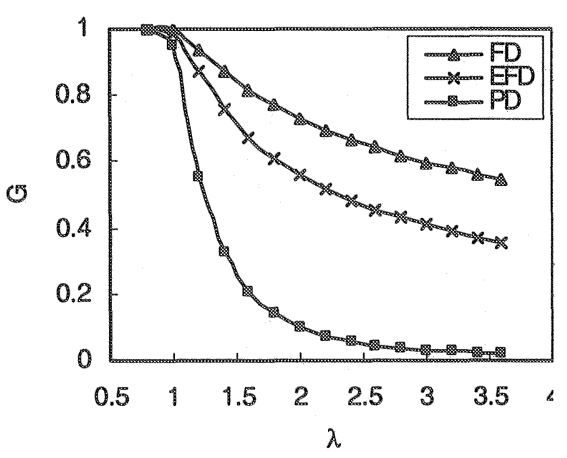

(b)

Figure 6. Good-throughput $\mathrm{G}$ vs. load per wavelength $\lambda$ for $\mathrm{FD}, \mathrm{EFD}$ and $\mathrm{PD}$ routers, $\mathrm{n}_{\mathrm{W}}=4, \mu=1, \mathrm{~L}=10, \mathrm{~B}=60$, and $\mathrm{k}=30$, (a) $\mathrm{q}=1 / 5$; (b) $\mathrm{q}=1 / 8$. 


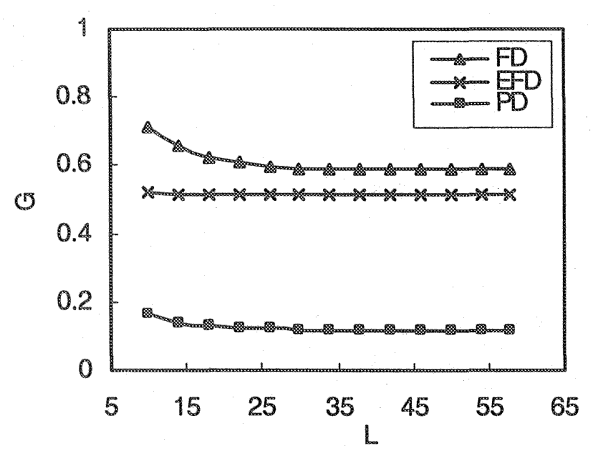

(a)

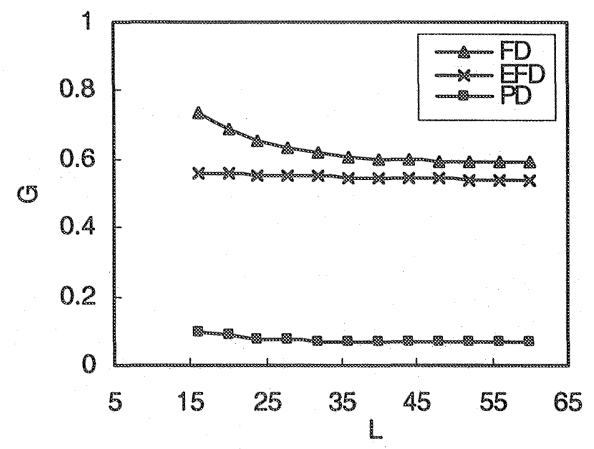

(b)

Figure 7. Good-throughput $\mathrm{G}$ vs. maximum number of packets $\mathrm{L}$ for $\mathrm{FD}, \mathrm{EFD}$ and $\mathrm{PD}$ routers, $\mathrm{n}_{W}=4, \lambda=2, \mu=1, \mathrm{~B}=60$, and $\mathrm{k}=30$, (a) $\mathrm{q}=1 / 5$; (b) $\mathrm{q}=1 / 8$.

\subsection{Effect of flow length}

Next the effect of the maximum number of packets $L$ on the goodthroughput for routers with 4 wavelength conversions is reported. As can be seen in Figs. 9a and 9b the reduction of G for FD is the largest among the three schemes. Saturation points exist for all the cases.

Finally the success probability Ps for flows with different number of packets is studied. As depicted in Fig. 10, the characteristic of Ps is similar to that in the buffer-only case [7]. For EFD routers, all the flows with different lengths have the same $\mathrm{Ps}$, since EFD routers accept flows regardless of their length. While for FD and PD routers, the shorter ones can obtain higher Ps than the longer ones. These results confirm that EFD routers are still the fairest one among the three. 


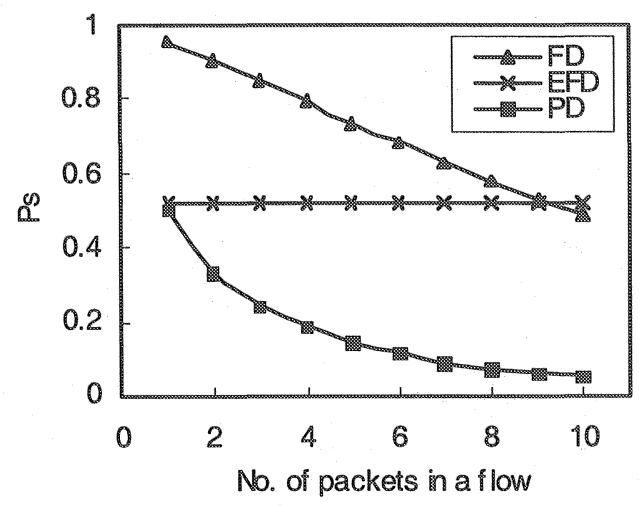

Figure 8. Flow success probability Ps vs. the number of packets in a flow for FD, EFD and PD routers, $\lambda=2, \mu=1, q=1 / 5, L=10, B=60, k=30$ and $n_{W}=4$.

\section{CONCLUSIONS}

Optical flow routers with wavelength conversion and optical buffering have been studied in order to improve the quality of the transmitted traffic in optical networks. A novel analytical model has been applied to evaluate the performance of wavelength-converted optical flow routers. The results have been compared with optical packet routers. It is shown that the application of wavelength conversion leads to the substantially increased good-throughput in optical flow routers. Up to $99 \%$ good-throughput can be achieved with 10 wavelength conversions and 60 buffers in optical flow routers with flow discard. As a result, $97 \%$ packets will be received in sequence after traffic goes through 3 wavelength-converted optical flow routers, comparing with only $8.4 \%$ obtained with 3 buffer-only optical flow routers. In addition, to achieve larger good-throughput the threshold of the optical flow routers with early-flow discard should be set as large as possible. On the other hand, it has been shown that the use of wavelength conversion cannot improve the good-throughput of optical packet routers, despite that the packet loss probability can be reduced by wavelength conversion. Our work strongly suggests that, in order to provide an application-friendly transmission environment for the future Internet, optical flow routers with wavelength conversion and optical buffering should be used instead of optical packet routers. 


\section{REFERENCES}

[1] S. A. Thomas, Ipng and the TCP/IP protocols implementing the next generation Internet, John Wiley \& Sons, Inc., 1996.

[2] M. Renaud, F. Masetti, C. Guillemot and B. Bostica, "Network and system concepts for optical packet switching", IEEE Communications Magazine, April 1997, pp. 96-102.

[3] J. C. R. Bennett, C. Patridge and N. Shectman, "Packet reordering is not pathological network behavior", IEEE/ACM Trans. On Networking, 1999, vol. 7, pp. 789m798.

[4] J. J. He and D. Simeonidou, "A flow-routing approach for optical IP networks", in Proc. of $O F C$ '2001, paper MN2.

[5] R. A. Barry and P. A. Humblet, "Models of blocking probability in all-optical networks with and without wavelength changers", IEEE Journal on Selected Areas in Communications, 1996, Vol. 14, pp. 858-867.

[6] S. L. Danielsen, C. Joergensen, B. Mikkelsen, and K. E. Stubkjaer, "Analysis of a WDM packet switch with improved performance under bursty traffic conditions due to tuneable wavelength converters", Journal of Lightwave Technology, 1998, vol. 16, pp. 729-735.

[7] J. J. He and D. Simeonidou, "Flow routing and its performance analysis in optical IP networks", Photonic Network Communications, vol. 3, pp. 49-62, 2001.

[8] J. J. He and D. Simeonidou, "Optical flow router performance: under ON-OFF traffic process”, in Proc. of NOC'2001, pp. 330-336. 